body). Endogenous endophthalmitis accounts for less than $15 \%$ of all cases of endophthalmitis and usually occurs in patients with other predisposing conditions, such as diabetes mellitus. ${ }^{1}$ In addition, patients receiving immunosuppressive drugs, persons abusing IV drugs, and patients undergoing invasive procedures are at increased risk for endophthalmitis. The usual primary foci of infection in endogenous endophthalmitis are meningitis, endocarditis, urinary tract infection, and wound infection.

The organisms responsible for endogenous endophthalmitis are varied. Fungal infections account for more than $50 \%$ of cases, among which Candida albicans is the commonest. The maximum concentration of organisms in endogenous endophthalmitis is believed to be in and around ocular blood vessels, which accounts for the efficacy of IV antibiotics. ${ }^{2}$

The clinical presentation of patients with endogenous endophthalmitis is variable, depending on the virulence of the organism. Mild cases are characterized by white nodules on the iris, retina, and choroid, whereas severe cases are characterized by opacification of the media and high intraocular pressures. Diabetic patients with wound infections are particularly susceptible to endophthalmitis. ${ }^{3}$

The systemic evaluation of a patient with suspected endogenous endophthalmitis includes a thorough history and physical examination to locate the extraocular source of infection. Ophthalmic ultrasonography can be useful in diagnosis. Specimens from both the anterior chamber and vitreous should be cultured because of potentially low yield in culturing organisms in a partially treated condition. The differential diagnosis includes conjunctivitis, glaucoma, and uveitis, among others, and these need to be excluded.
Our patient had diabetes mellitus, underwent a major operation, and then had a wound infection. This placed him at increased risk for septicemia and endogenous endophthalmitis. The sternal wound was culture positive for Staphylococcus aureus, but no organisms were identified in the ocular cultures, perhaps because IV antibiotics were begun 4 days before the diagnosis of endogenous endophthalmitis. Organisms are identified in about two thirds of patients with clinically diagnosed endophthalmitis.

The treatment of the patient with endogenous endophthalmitis consists of systemic and intravitreal antibiotics as soon as the diagnosis is made. The role of vitrectomy is complex. Its purpose is to obtain a good culture specimen, to remove toxins and cells, and to create space for intravitreal antibiotics.

In conclusion, if a patient has eye pain or visual disturbance after a cardiac operation and if he or she has a known source of infection, the diagnosis of endophthalmitis should be considered.

\section{REFERENCES}

1. Okada AA, Johnson RP, Liles WC, D'Amico DJ, Baker AS. Endogenous bacterial endophthalmitis: report of a ten-year retrospective study. Ophthalmology 1994;101:832-8.

2. Endophthalmitis Vitrectomy Study Group. Results of the endophthalmitis vitrectomy study. Arch Ophthalmol 1995;113: 1479-96.

3. Phillips WB, Tasman WS. Postoperative endophthalmitis in association with diabetes mellitus. Ophthalmology 1994;101: $508-18$.

\title{
ELECTROSURGERY WITH A FIBEROPTIC BRONCHOSCOPE AND A SNARE FOR ENDOTRACHEAL/ENDOBRONCHIAL TUMORS
}

Motoyasu Sagawa, MD, Masami Sato, MD, Hiroto Takahashi, MD, Muneo Minowa, MD, Yasuki Saito, MD, and Shigefumi Fujimura, MD, Sendai, Japan

Endobronchial tumors sometimes cause stenosis of the major bronchi, which requires prompt palliation. A num-

From the Department of Thoracic Surgery, Institute of Development, Aging and Cancer, Tohoku University, Sendai, Japan.

Received for publication Jan. 2, 1998; accepted for publication Feb. 13, 1998

Address for reprints: Motoyasu Sagawa, MD, Department of Thoracic Surgery, Institute of Development, Aging and Cancer, Tohoku University, 4-1 Seiryo-machi, Aoba-ku, Sendai 980-77, Japan.

J Thorac Cardiovasc Surg 1998;116:177-9

Copyright (C) 1998 by Mosby, Inc.

0022-5223/98 $\$ 5.00+0 \quad \mathbf{1 2 / 5 4 / 8 9 6 6 7}$ ber of techniques for palliation have been reported, such as the neodymium-yttrium-aluminum-garnet (Nd-YAG) laser, ${ }^{1}$ endoscopic cryosurgery, ${ }^{1,2}$ and electrosurgery with a rigid bronchoscope. ${ }^{1,3}$ However, electrosurgery with a fiberoptic bronchoscope and a snare is not a widely known technique in the surgery of the respiratory tract. ${ }^{4,5}$ In this study, we evaluate the effectiveness and complications of the technique in nine cases of tracheobronchial tumors resected by this technique. The indications and limitations of the technique are also discussed.

Technique. Endobronchial electrosurgery was performed under local anesthesia with an electrosurgical unit (UES/UES-10, Olympus, Tokyo, Japan), a flexible fiberoptic bronchoscope (BF-1TR/BF-P30/BF-P240, Olympus), and a snare (SD-11L-1/SD-18C-1, Olympus). Oxy- 
Table I. The summary of the cases

\begin{tabular}{ccclccccc}
\hline Patient & $\begin{array}{c}\text { Age } \\
(y r)\end{array}$ & Sex & \multicolumn{1}{c}{ Location } & \multicolumn{1}{c}{ Histology } & Stenosis & Aim of electrosurgery & Result & Complication \\
\hline 1 & 74 & F & Trachea & Adenocarcinoma & $95 \%$ & Dilation of stenosis & Success & None \\
2 & 54 & F & Trachea & Squamous papilloma & No & Curative resection & Success & None \\
3 & 60 & M & Trachea & Squamous cell carcinoma & $95 \%$ & Dilation of stenosis & Failure & Dyspnea \\
4 & 75 & M & Left main bronchus & Squamous cell carcinoma & $100 \%$ & Evaluation of invasion & Success & None \\
5 & 24 & F & Left main bronchus & Hamartoma & $95 \%$ & Dilation of stenosis & Success & None \\
& & & & & Curative resection & Success & None \\
6 & 75 & M & Left main bronchus & Adenocarcinoma & $90 \%$ & Dilation of stenosis & Success & None \\
7 & 30 & F & Intermediate trunk & Neurinoma & $100 \%$ & Dilation of stenosis & Success & None \\
& & & & & Curative resection & Failure & None \\
8 & 77 & M & Left lower bronchus & Squamous cell carcinoma & $95 \%$ & Dilation of stenosis & Success & None \\
9 & 65 & M & Left basal bronchus & Large-cell carcinoma & $90 \%$ & Dilation of stenosis & Success & None
\end{tabular}

gen was given with nasal prongs. Patients received pentazocine, hydroxyzine hydrochloride, and atropine sulfate before the operation. Arterial oxygen saturation, blood pressure, and the electrocardiogram were monitored during the electrosurgery. An endotracheal tube was placed unless the stenosis was located in the upper part of the trachea. Careful manipulation with a snare was required to lasso the polypoid tumor. Energy at $40 \mathrm{~W}$ was applied, and the snare was pulled very slowly to avoid causing a mechanical cut by the snare that would produce bleeding. After the polypectomy, the resected specimen was removed with a grasping forceps.

Patients. Patient characteristics and results are summarized in Table I. The patients included five men and four women, having six malignant and three benign tumors. The tracheobronchial stenosis was severe in all the patients except one who had a squamous papilloma. The aims of the electrosurgery were dilation of the tracheobronchial stenosis in seven patients, curative resection of a benign tumor in three patients, and evaluation of carcinoma invasion in one patient.

Effects. The stenotic area was successfully dilated in six (patients 1, 5, 6, 7, 8, and 9) of the seven patients in whom this was the aim. In the remaining patient (patient 3 ), an emergency tracheostomy was performed for increased dyspnea.

In two (patients 2 and 5) of three patients with a benign tumor, curative resection of the tumor was successful and an additional thoracotomy was not required. In the remaining patient (patient 7), most of the tumor was removed by electrosurgery and the atelectasis of the right middle and lower lobes was relieved. However, a part of the tumor could not be removed and the atelectasis of the right $S^{6}$ segment (the superior segment of the lower lobe) remained. A sleeve $\mathrm{S}^{6}$ segmentectomy was performed later.

Carcinoma invasion was successfully evaluated in one patient (patient 4). The protruding part of the tumor was resected without bleeding.

Intraoperative condition of the patients and complications. In eight of nine patients, electrosurgery was performed within 10 minutes, taking only a few minutes in most of them. This technique caused little smoke, and the arterial oxygen saturation, blood pressure, and electrocardiogram were not changed during the operation. Patients' symptoms, such as dyspnea, coughing, and stridor, were relieved promptly. No additional complications were observed.

Lassoing the tumor with a snare was very difficult in patient 3, and the patient's dyspnea was worsened. An emergency tracheostomy was required.

Discussion. A number of techniques have been reported for the palliation of bronchial obstruction or stenosis. The Nd-YAG laser is one of the most useful devices for dilatation of the stenotic bronchial tree. ${ }^{1}$ However, Nd-YAG laser therapy causes smoke, which sometimes decreases the arterial oxygen saturation of the patient and takes a relatively long time. Electrosurgery with a rigid bronchoscope is also a useful technique, ${ }^{1,3}$ but it requires general anesthesia and only limited tumor sites can be reached. Endoscopic cryosurgery is a safe technique because the bronchial cartilage cannot be damaged, ${ }^{1,2}$ but effective dilation with this technique requires a long time. Brachytherapy ${ }^{1}$ needs much time, too. These two techniques cannot be applied to patients who need prompt relief of dyspnea.

Electrosurgery with a fiberoptic bronchoscope and a snare caused little smoke, required only local anesthesia, and took only a few minutes to resect the tumor. This technique can be applied to polypoid tumors located anywhere within the sight of the bronchoscope. Patients' symptoms, including dyspnea, coughing, and stridor, were relieved promptly. A large specimen obtained by the technique made histologic examination easier. In some benign tumors, additional thoracotomy was not required.

This technique had some limitations. In patient 7 , dilation of the intermediate trunk was successful, but dilation of the $\mathrm{B}^{6}$ segmental bronchus was not. The tumor at the intermediate trunk was polypoid, but the remaining tumor at the $\mathrm{B}^{6}$ segmental bronchus was not. This technique can be applied only to polypoid tumors. In patient 3 , resection of the tracheal tumor was unsuccessful. The tumor was located so close to the vocal cord that handling a fiberoptic bronchoscope and a snare was difficult. This case was our first attempt with this technique and that may 
have influenced the result. Recently, a new snare for the respiratory tract has become available, and a similar operation was successfully done more easily in similar a case (patient 1).

Although electrosurgery with a fiberoptic bronchoscope and a snare has some limitations, it is very useful and may be the first choice for the resection of polypoid tumors in the bronchial tree.

We are grateful to Ms. Chieko Yoshida, Mr. Toshihiko Kanno, Ms. Mieko Kosuga, and Ms. Mutsuko Izumi for their technical assistance.

\section{REFERENCES}

1. Jacobson MJ, LoCicero J. Endobronchial treatment of lung carcinoma. Chest 1991;100:837-41.

2. Marasso A, Gallo E, Massaglia GM, Onoscuri M, Bernardi V. Cryosurgery in bronchoscopic treatment of tracheobronchial stenosis. Chest 1993;103:472-4.

3. Petrou M, Goldstraw P. The management of tracheobronchial obstruction: a review of endoscopic technique. Eur J Cardiothorac Surg 1994;8:436-41.

4. Themelin D, Duchatelet P, Boudaka W, Lamy V. Endoscopic resection of an endobronchial hypernephroma metastasis using polypectomy snare. Eur Respir J 1990;3:732-3.

5. Gerasin VA, Shafirovsky BB. Endoscopic electrosurgery. Chest 1988;93:270-4.

\section{CATAMENIAL PNEUMOTHORAX CAUSED BY DIAPHRAGMATIC ENDOMETRIOSIS}

S. Blanco, PhD, F. Hernando, PhD, A. Gómez, PhD, M. J. González, MD, A. J. Torres, PhD, and J. L. Balibrea, PhD, Madrid, Spain

Catamenial pneumothorax is a recurrent syndrome of pneumothorax that occurs between 48 and 72 hours after menstruation. This syndrome was described for the first time in $1958^{1}$ and was named "catamenial pneumothorax" by Lillington in 1972. Since then more than 100 cases have been described in the literature. ${ }^{2}$ Considered a rare complication of systemic endometriosis, catamenial pneumothorax has been managed with a wide range of treatments ranging from thoracotomy with abrasive or chemical pleurodesis ${ }^{2}$ to the present-day treatment with oral contraceptives, progesterone, or gonadotropin-releasing hormone analogs. ${ }^{3}$

The causes of catamenial pneumothorax are diverse. Although most episodes are associated with diaphragmatic endometriosis, few have been confirmed in anatomopathologic studies. ${ }^{2,4,5}$ This report presents a case of catamenial pneumothorax in which systemic endometriosis was confirmed histologically.

Clinical summary. A 33-year-old woman had a history of chest pain associated with menstruation. In November 1992 she had an episode of right-sided pneumothorax that resolved with rest. In September 1995, she had chest pain on the right side with sudden dyspnea. Chest radiography revealed hydropneumothorax with complete collapse of the

From the Department of Surgery, Thoracic Surgery, Hospital Clinico "San Carlos," Madrid, Spain.

Received for publication Dec. 9, 1997; accepted for publication Jan. 9, 1998.

Address for reprints: S. Blanco, MD, Cirugia II y Cirugia Toracica, Hospital Clinico "San Carlos," C/Martin Lagos S/N, 28040, Madrid, Spain.

J Thorac Cardiovasc Surg 1998;116:179-80

Copyright (c) 1998 by Mosby, Inc.

0022-5223/98 $\$ 5.00+0 \quad \mathbf{1 2 / 5 4 / 8 8 8 1 9}$ lung. Endothoracic drainage resulted in complete reexpansion of the lung. On thoracic computed tomography no bullae were observed with pleural nodular swelling. Nuclear magnetic resonance imaging revealed an image in the pleura in the posterior arches of the fifth and sixth ribs of the right hemithorax, which could correspond to a blood focus or endometriosis. Abdominal ultrasonography did not reveal any pathologic findings. Intervention with videothoracoscopy was elected and bullae were detected in the apical segment of the upper lobe and in the posterior segment of the right lower lobe, which were resected. Mechanical pleural abrasion was performed. Exploration of the rest of the thoracic cavity revealed no associated lesions. Anatomopathologic study gave a diagnosis of bullous emphysema. In conjunction with the next menstrual cycle the patient again had severe chest pain on the right side and pleuritic signs. Chest radiography revealed apical and basal pneumothorax. On suspicion of catamenial pneumothorax a right axillary thoracotomy was performed in November 1995 with resection of several suggestive lesions from all the lobes and of a purple lesion $0.5 \mathrm{~cm}$ diameter from the right diaphragmatic cupula. Partial pleurectomy and pleural abrasion were again performed. Definitive anatomopathologic study revealed diaphragmatic endometriosis (Fig. 1). The patient was treated with gonadotropin-releasing hormone analogs because she wants to have children in the future. She is currently free of symptoms after 12 months of follow-up.

Discussion. Catamenial pneumothorax occurring in the few hours after menstruation accounts for $2.8 \%$ to $5.6 \%$ of all episodes of spontaneous pneumothorax in women. Usually numerous episodes of pneumothorax are necessary before the condition can be diagnosed. ${ }^{2}$ Most women affected with catamenial pneumothorax are in the third or fourth decade of their lives ${ }^{2,4,5}$ and the right hemithorax is affected in $90 \%$ to $95 \%$ of cases. ${ }^{2,4,5}$ The most frequent 